International Journal of Distributed and Parallel Systems (IJDPS) Vol.3, No.1, January 2012

\title{
BLUETOOTH SECURITY THREATS AND SOLUTIONS: A SURVEY
}

\author{
Nateq Be-Nazir Ibn Minar ${ }^{1}$ and Mohammed Tarique ${ }^{2}$ \\ ${ }^{1}$ Department of Electrical and Electronic Engineering, American International \\ University, Bangladesh \\ nateqminareieee.org \\ ${ }^{2}$ Department of Electronic and Communication, Ajman University of Science and \\ Technology, United Arab Emirates \\ m.tarique@ajman.ac.ae
}

\begin{abstract}
Bluetooth technology has become an integral part of this modern society. The availability of mobile phones, game controllers, Personal Digital Assistant (PDA) and personal computers has made Bluetooth a popular technology for short range wireless communication. However, as the Bluetooth technology becomes widespread, vulnerabilities in its security protocols are increasing which can be potentially dangerous to the privacy of a user's personal information. The security issues of Bluetooth have been an active area of research for the last few years. This paper presents the vulnerabilities in the security protocols of this technology along with some past security threats and possible countermeasures as reported in the literatures which have been surveyed and summarized in this paper. It also presents some tips that end-users can implement immediately to become more cautious about their private information. Finally, the paper concludes with some recommendations for future security enhancements that can be implemented in the Bluetooth standard.
\end{abstract}

\section{KEYWORDS}

Bluetooth, encryption, security protocols, security threats, countermeasures, Bluetooth enhancements

\section{INTRODUCTION}

Bluetooth technology has been considered as a cheap, reliable, and power efficient replacement of cables for connecting electronic devices. This technology was officially approved in the summer of 1999 [1]. Since then it has widely been used in various electronic devices. Bluetooth Special Interest Group (SIG) was formed to nurture and promote this technology. The SIG has over 14,000 members including some leading companies in the fields of telecommunications, computing, automotive, music, industrial automation, and network industries [2]. Bluetooth is a combination of hardware and software technology. The hardware is riding on a radio chip. On the other hand, the main control and security protocols have been implemented in the software. By using both hardware and software Bluetooth has become a smart technology for efficient and flexible wireless communication system. Bluetooth radio chip supports communication among a group of electronic devices. Once the hardware radio chips are installed into the 
electronic devices, wireless communication can be established among these devices. The operating distance between two Bluetooth devices ranges from 10 and 100 meters. By using a directional antenna and an amplifier the range of Bluetooth can be extended over a mile away. One of the major advantages of Bluetooth technology is that it operates in a license-free Industrial, Scientific and Medical (ISM) band ranging from 2.4 to $2.4835 \mathrm{MHz}$. This band is divided into 79 channels each being $1 \mathrm{MHz}$ wide. Using Fast Frequency Hopping Sequence (FFHS) a Bluetooth device hops from one channel to another channel up to 1600 times in one second [9]. Bluetooth also uses Adaptive Frequency Hopping (AFH) technique which is designed to cope with excessive packet losses due to packet collisions or external interferences. Each Bluetooth chip has a unique identity code. The 'master-slave' concept is the core of a Bluetooth based network [5]. The 'master' works as the moderator during the communication between itself and the slave as well as among the slaves themselves.

In Bluetooth a trusted relationship between two devices called 'pairing' are formed by exchanging shared secret codes referred to as PINs. A 'master' device has the option of pairing with up to seven 'slave' devices establishing a network called a piconet. Two or more piconets together form a scatternet, which can be used to eliminate Bluetooth range restrictions. A scatternet is formed when the devices act as 'master' or 'slave' devices in multiple piconets at the same time. A more detail description of Bluetooth technology can be found in [4]. A summary of the other key features of Bluetooth technology has been presented in Table-1.

Table 1. Bluetooth Technical Specification

\begin{tabular}{|l|l|}
\hline Connection & Spread Spectrum(Frequency \\
\hline Frequency band & $2.4 \mathrm{GHZ}$ ISM \\
\hline Modulation Technique & Gaussian Frequency Shift \\
\hline MAC Scheduling scheme & FH-CDMA \\
\hline Transmission Power & $>20 \mathrm{dBm}$ \\
\hline Aggregate Data Rate & $0.721-1 \mathrm{Mbps}$ \\
\hline Range & $10 \mathrm{~m}-100 \mathrm{~m}$ \\
\hline Supported Stations & 8 devices (per Piconet) \\
\hline Voice Channels & 3 \\
\hline Data Security- & 128 bit key \\
\hline Data Security-Encryption & $8-128$ bits(configurable) \\
\hline
\end{tabular}

With each release of a new Bluetooth version, the manufacturers have upgraded different aspects of this technology to make it more secure and user-friendly to support a wide range of devices, a list of all the Bluetooth versions released to date is mentioned in [12]. The last version to be released was version 4.0 which had the most versatile design and was focused on low power usage [13]. Although the Bluetooth technology is undoubtedly considered a very popular technology, it has some security 'loop-holes' that make it vulnerable. In this paper, these vulnerability issues have been addressed. The security threats and solutions proposed in the literatures have been surveyed and summarized in this paper. The rest of the paper is organized as follows, Section II describes some related work done with Bluetooth security protocols and Section III explains the Bluetooth protocol stacks. The security architecture of Bluetooth technology has been explained in section IV. Section V contains the vulnerabilities 
of this technology. The security threats reported so far in the literatures have been complied in section VI. Counter measures against the security threats have been presented in section VII and section VIII presents some security tips for the users to create awareness among them to protect their private information while communicating, to mitigate the risks of being attacked. The paper is concluded with section IX.

\section{RELATED WORK}

Many security experts in the field of wireless technologies have conducted research on different aspects within the security architecture of Bluetooth and have provided amazing results with new tweaks that enhances the security of the device within a network. Some commendable research work is mentioned in [6]. [7] and [8].

In [6], the authors have presented a light weight protocol to provide location privacy in wireless body area network. The basic idea of their protocol is on the use of temporary pseudonyms instead the use of hardware addresses to communicate in the wireless body area networks. This allows protecting the source and the destination of mobile devices in the WBANs. Their protocol is efficient and also energy saving.

In [7], the authors proposed the design of a device pairing simulator called "PSim", they have felt the need to create this tool because most wireless systems are prone to security risks, such as eavesdropping and require different techniques as compared to traditional security mechanisms to test their security protocols. This tool can be used to perform test on different types of device pairing methods as well as generate new protocols for increased security measures.

In [8], the authors have compared different techniques used for device pairing in wireless networks and have presented a comparative result of their findings on the security protocols used.

Besides the work mentioned here, there are other numerous papers published and research work done which are beyond the scope of this paper to elaborate on all of them, but they all aim to improve wireless network security systems and since Bluetooth is a common wireless standard among almost all devices, its security must be given a high priority due to its widespread usage.

\section{BLUetooth Protocol Stacks}

A protocol stack is a combination of software/hardware implementation of the actual protocols specified in the standard [11]. It also defines how the devices should communicate with each other based on the standard. The Bluetooth protocol stack is shown in Fig. 1. 


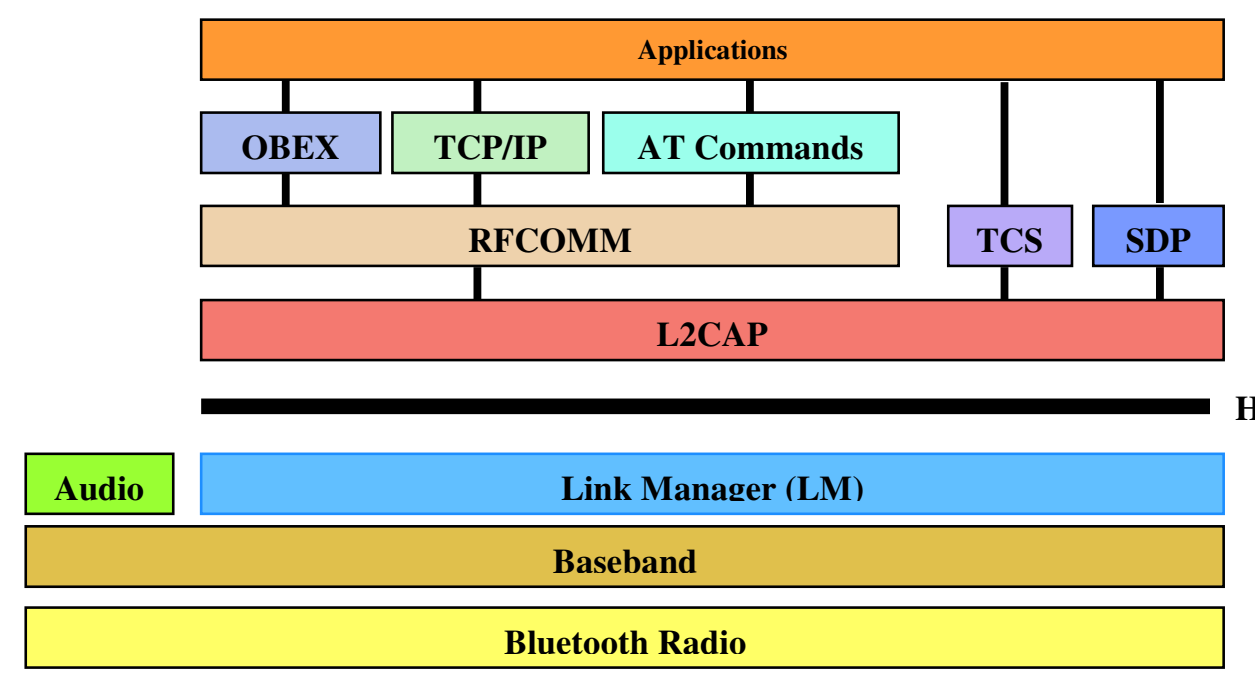

Application specific security protocols

\section{Bluetooth host security protocols}

\section{HCI}

\section{Security protocols on Bluetooth hardware chip}

Fig. 1 Bluetooth Protocol Stack

The protocols below the host controller interface (HCI) are built into the Bluetooth microchip and the protocols above the HCI are included in the host device's software package. The HCI ensures a secured communication between the host and the Bluetooth module. The radio layer transmits data in the form of bits by using a radio frequency. This function is defined by the radio layer. Bluetooth transceivers use Gaussian Frequency Shift Keying (GFSK) technique. The baseband layer performs the functions of frequency hopping for interference mitigation, medium access control and forming data packet. In addition, the baseband layer also controls link, channel, error correction and flow control. It establishes two kinds of link depending on the application and operating environment.

A synchronous connection oriented (SCO) link is established to emulate circuit switched connections for voice and data connection. While an asynchronous connection link (ACL) is defined for the data bursts. This link also supports broadcasting and data rate control by the master device. The link manager (LM) acts as a liaison between the application and the link controller (LC) on the local device. It is also used for communication with the remote LM via protocol data units (PDU) and the link manager protocol (LMP). The audio protocol is used for a real time two way voice communication. The audio protocol is carefully located in such a way so that the overhead of upper layer protocols does not cause any delays for real-time twoway voice connections.

The logical link control and adaptation protocol (L2CAP) is a software module that normally resides in the host. It acts as a conduit for data on the asynchronous connection link (ACL) between the baseband and host applications. The L2CAP is used to ensure both connection oriented and connection less services. Connection oriented service is used for communication between the master to one slave. Connection less service is used for communication between a master and multiple slaves. The L2CAP can initiate security procedures when a connection oriented or a connectionless connection request is made.

The Object Exchange Protocol (OBEX) is used to exchange objects such as calendar notes, business cards and data files between devices based on a client-server model. The telephony control specification (TCS) defines the call control signaling for the establishment/release of 
speech and data calls between Bluetooth devices. It also provides functionality for exchanging signaling information not related to ongoing calls.

The service discovery protocol (SDP) discovers the services that are available in the RF proximity and determines the characteristic of these available services. SDP is an essential protocol that enables the Bluetooth devices to form an ad hoc network. RFCOMM is a transport protocol used to emulate the RS-232 serial ports. This protocol enables a Bluetooth device to connect with external devices like printers and scanners. The RFCOMM protocol relies on the baseband protocol stack to provide reliable in-sequence delivery of bit stream.

\section{SECURITY ARCHITECTURE}

Security issues have played a major role in the invention of Bluetooth technology. The Bluetooth SIG has put much effort into making Bluetooth a secured technology. Several security measures have been implemented at different protocol levels, but the basic Bluetooth security configuration depends on the user's Bluetooth device, who decides about the discoverability and connection options. In general, Bluetooth discoverability and connection options are divided into three 'modes' of operation [14], which are as follows:

- Silent: The device will never accept any connections. It simply monitors the Bluetooth traffic.

- Private: The device cannot be discovered. A connection will be accepted only if the Bluetooth device address (BD_ADDR) of the device is known to the prospective master. A 48-bit BD_ADDR is normally unique and it refers globally to only one individual Bluetooth device.

- Public: The device can be both discovered and connected to. It is, therefore, called a discoverable device.

In addition to these modes, there are also four different security modes that a device can implement. These are as follows-

- Non-secure: The Bluetooth device does not initiate any security measures.

- Service-level enforced security mode: Two Bluetooth devices can establish a nonsecure ACL. Security procedures are initiated after an L2CAP connection oriented or an L2CAP connection-less channel request is made.

- Link-level enforced security mode: Security procedures are initiated when an ACL link is established and before any channel request is made.

- Service-level enforced security mode (SSP): This mode is similar to mode 2, except that only Bluetooth devices using secure simple pairing (SSP) can use it.

There are three main steps in Bluetooth security procedures, which are as follows

- Authentication: It involves proving the identity of one Piconet device to another. The objective of the authentication procedure is to determine the client's authorization level. The authentication is verified by checking the link keys. The sender encrypts the Bluetooth device address of the receiver using the link key and a random number to produce a signed response authentication result (SRES). The SRES is sent to the receiver and the connection is established if the two link keys are equal.

- Authorization: It is the process of granting or denying access to a network resource. 
- Optional Encryption: It is the encoding of information being exchanged between Bluetooth devices in a way that eavesdroppers cannot decode its contents. The encryption is an essential part of Bluetooth security. The encryption key can vary between 8 and 128 bits. The user does not have access to change the size of the encryption key as the key size must be specified by the manufacturers according to the countries' regulations. A random number must be sent from one device to the other when any two Bluetooth devices wish to start the communication. The receiving device must also have knowledge of the PIN from the sending devices. With these two sets of information, a link key is generated on both devices.

Bluetooth security is based on building a chain of events. None of these events provides any meaningful information to an eavesdropper. All the events must occur in a specific sequence for the enforcement of secured communication between two Bluetooth enabled devices. Two Bluetooth devices begin pairing with the same PIN code that is used for generating several 128bit keys. The same PIN code can be used for all Bluetooth enabled devices in a trusted network. For example, in a personal Bluetooth network environment consisting of various Bluetooth devices such as a mobile phone, a printer, and a DVD player the same PIN code can be used. However, each master-slave pair can also have a different PIN code for providing trusted relationship between each pair of devices. For example, in a conference environment where two people meet for the first time and they want to create a Bluetooth network between their electronic devices, the PIN selection should be done by using a different PIN codes for that master-slave pair. Otherwise all other Bluetooth connections that are using the same PIN code may be compromised. Fig. 2 shows the detailed pairing process of two Bluetooth enabled devices. 


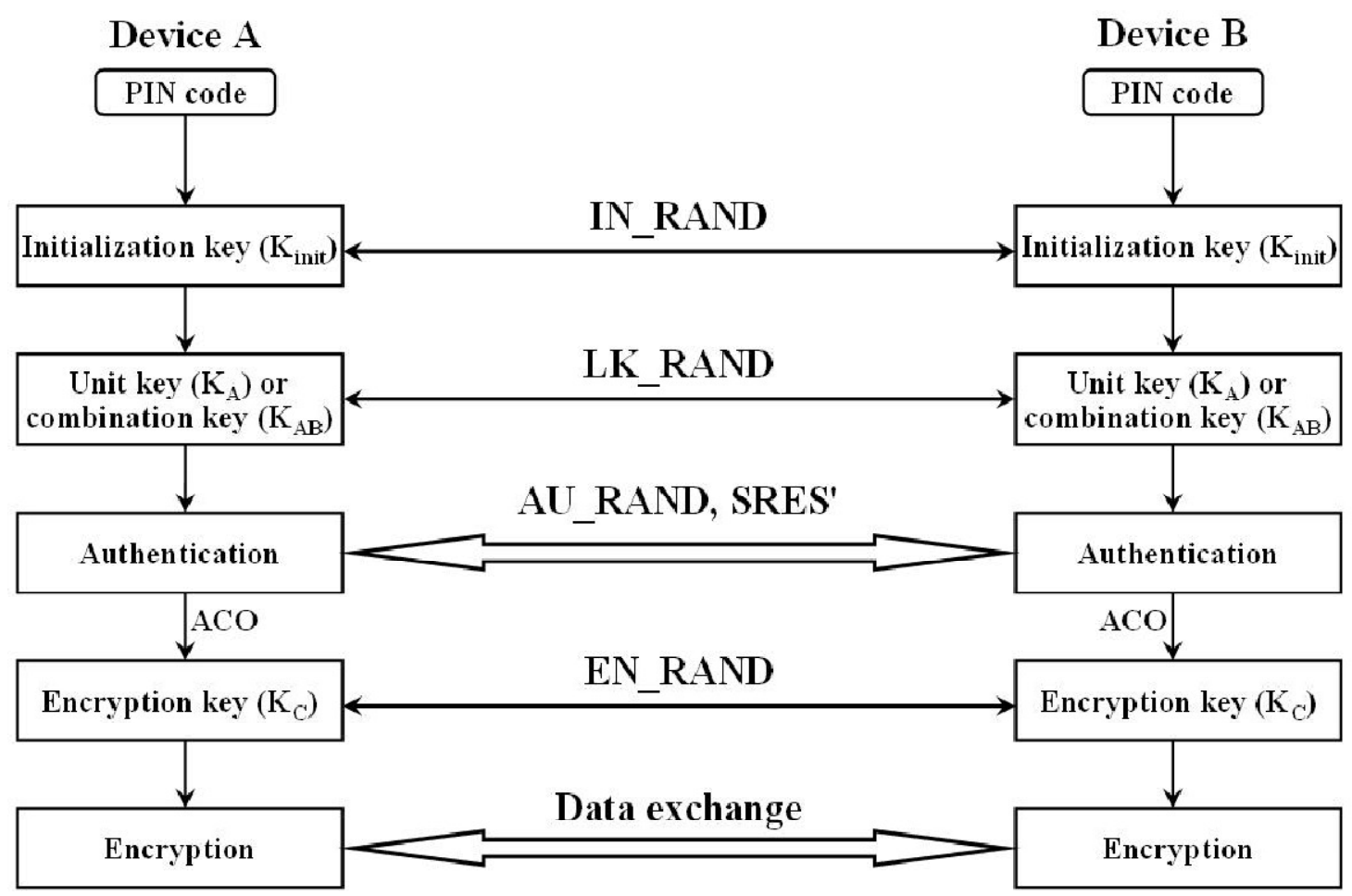

Fig. 2 Illustration of Bluetooth security operations

An initialization key $\left(\mathrm{K}_{\text {init }}\right)$ is generated when two Bluetooth devices meet for the first time and it is used for generating more secured 128-bit keys, which are generated during the next phases of the security chain of events. The $\mathrm{K}_{\text {init }}$ is derived from a 128-bit pseudorandom number IN_RAND, an L-byte $(1 \leq \mathrm{L} \leq 16)$ PIN code, and the BD_ADDR. It is worth noting to mention that the IN_RAND is sent via air in unencrypted form. The $\mathrm{K}_{\text {init }}$ is produced in both devices using $\mathrm{K}_{\text {init }}=\mathrm{E}_{22}(\mathrm{PIN}$ ',L',IN_RAND). The PIN code and its length L are modified into two different quantities called 'PIN' and L' before sending them to the $\mathrm{E}_{22}$ function. If the PIN is less than 16 bytes, it is augmented by appending bytes from the device's BD_ADDR until the 'PIN' either reaches a total length of 16 bytes or the entire BD_ADDR is appended, whichever comes first. If one device has a fixed PIN code, the BD_ADDR of the other device is used. If both devices can support a variable PIN code, the BD_ADDR of the device that received the IN_RAND is used. The $\mathrm{K}_{\text {init }}$ is used to encrypt a 128-bit pseudorandom number (LK_RAND), i.e. LK_RAND $\oplus \mathrm{K}_{\text {init }}$ is exchanged in the next phase of the security chain of events when a link key (a unit key or a combination key) is generated. A unit key $\left(\mathrm{K}_{\mathrm{A}}\right)$ is produced from the information of only one device (device $A$ ) using the formula $\mathrm{K}_{\mathrm{A}}=\mathrm{E}_{21}\left(\mathrm{BD}_{-} \mathrm{ADDR}_{\mathrm{A}}, \mathrm{RAND}_{\mathrm{A}}\right)$. Device $A$ encrypts the $K_{A}$ with the $K_{\text {init }}$ (i.e. $K_{A} \oplus K_{\text {init }}$ ) and sends it to device B. Device $B$ decrypts the $K_{A}$ with the $K_{\text {init }}$ by $\left(K_{A} \oplus K_{\text {init }}\right) \oplus K_{\text {init }}=K_{A}$. Now, the both devices have the same $K_{A}$ as a link key. Only devices that have limited resources to store several keys, these devices should use the unit key. And the security enforced by the unit key is only a low level of security. Therefore, Bluetooth specifications do not recommend using the unit key anymore.

A combination key $\left(\mathrm{K}_{\mathrm{AB}}\right)$ is dependent on two devices and therefore it is derived from the information of both devices. The $K_{A B}$ is produced in both devices using $K_{A B}=E_{21}\left(B D \_A D D R_{A}\right.$, 
LK_RAND $\left.{ }_{\mathrm{A}}\right) \oplus \mathrm{E}_{21}\left(\mathrm{BD} \_\mathrm{ADDR} \mathrm{B}_{\mathrm{B}}, \mathrm{LK}_{-} \mathrm{RAND} \mathrm{B}_{\mathrm{B}}\right)$. It is worth noting to mention that generating the $\mathrm{K}_{\mathrm{AB}}$ is nothing more than a simple bitwise XOR between two unit keys, i.e. $\mathrm{K}_{\mathrm{AB}}=\mathrm{K}_{\mathrm{A}} \oplus \mathrm{K}_{\mathrm{B}}$. Each device can produce its own unit key and each device also has the BD_ADDR of the other device. Therefore, two devices have to exchange only their respective pseudorandom numbers in order to produce each other's unit keys.

Device A encrypts the LK_RAND ${ }_{A}$ with the current key $K$ by LK_RAND $\oplus$ $\oplus K$, where $K$ can be the $K_{\text {init }}$, the $K_{A}$ or the $K_{A B}$ that was created earlier. Device $A$ then sends the key to device $B$. The $\mathrm{K}$ is the $\mathrm{K}_{\mathrm{init}}$ if the devices create a link key for the first time together. The $\mathrm{K}$ is the $\mathrm{K}_{\mathrm{A}}$ if the link key is a unit key, and it is the $\mathrm{K}_{\mathrm{AB}}$ if the link key is being upgraded to a combination key.

Device B decrypts the LK_RANDA with the K, (i.e., LK_RAND $\oplus$ $\oplus \mathrm{K} \oplus \mathrm{K}=\mathrm{LK} \_$RAND ${ }_{\mathrm{A}}$ ), and can now produce the $\mathrm{K}_{\mathrm{A}}$. Correspondingly, device $\mathrm{B}$ encrypts the $\mathrm{LK}_{-} \mathrm{RAND}_{\mathrm{B}}$ with the $\mathrm{K}$ (i.e., LK_RAND $\left.{ }_{B} \oplus K\right)$, and sends it to device A. Device A decrypts the LK_RAND ${ }_{B}$ with the K ( i.e., $\left.L K \_R A N D_{B} \oplus K \oplus K=L K \_R A N D_{B}\right)$, and produces the key $K_{B}$. Finally, both devices can produce the $\mathrm{K}_{\mathrm{AB}}$ by using $\mathrm{K}_{\mathrm{A}}$ and $\mathrm{K}_{\mathrm{B}}\left(\right.$ i.e., $\mathrm{K}_{\mathrm{AB}}=\mathrm{K}_{\mathrm{A}} \oplus \mathrm{K}_{\mathrm{B}}$ ).

The next phase of the security chain of events is the challenge response authentication in which a claimant's knowledge of a secret link key is checked as illustrated in Fig. 3. During each authentication, a new 128-bit pseudorandom number AU_RAND is exchanged via air in an unencrypted form. Other inputs to the authentication function $\mathrm{E}_{1}$ are the BD_ADDR of the claimant and the current link key $\left(\mathrm{K}_{\mathrm{A}}\right.$ or $\left.\mathrm{K}_{\mathrm{AB}}\right)$.

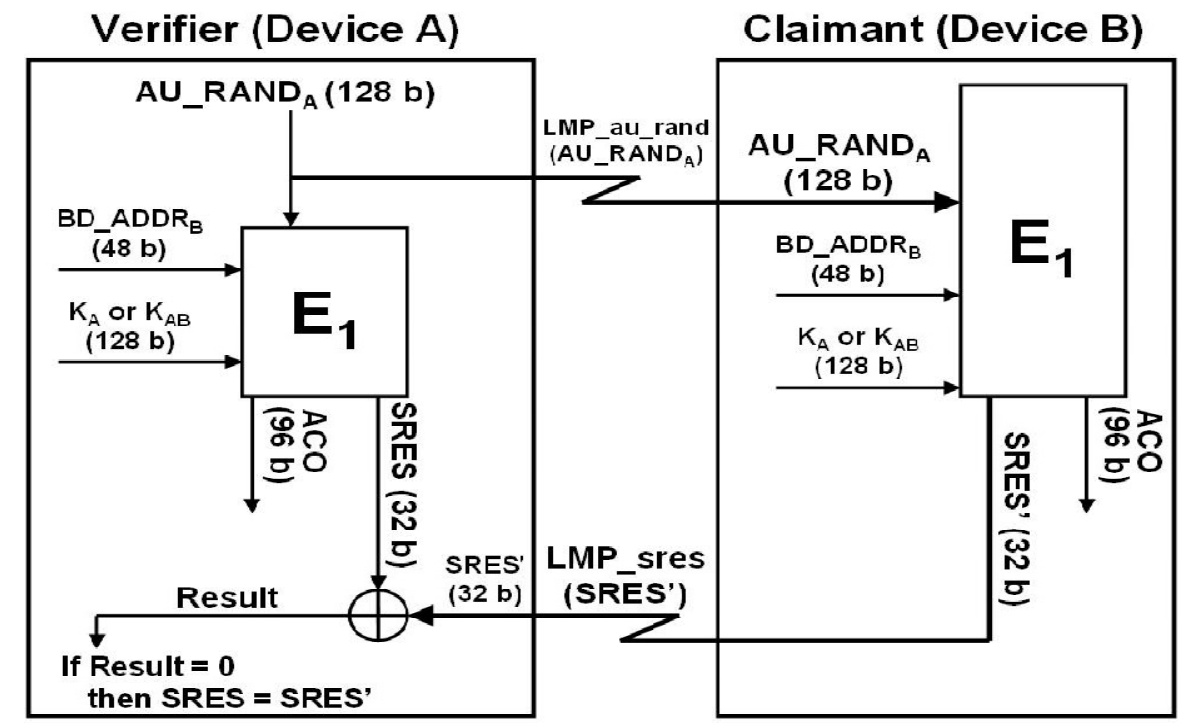

Fig. 3 Bluetooth challenge-response authentication

A 32-bit SRES and a 96-bit authenticated ciphering offset (ACO) are produced in both devices by $E_{1}\left(A U \_R A N D_{A}, B_{-}{ }_{2} D_{D D R}\right.$, Link key) function, where the Link key is the $K_{A}$ or the $K_{A B}$. The claimant sends the SRES' (i.e., the SRES value produced by the claimant), via air in unencrypted form to the verifier. The verifier compares the generated SRES value with the received SRES value, and if these values match with each other, the authentication is 
successfully completed. The ACO is used in the next phase of the security chain of events when an encryption key is generated.

It is worth noting to mention that the SRES and the SRES' are 32-bit numbers, not 128-bit numbers. The 32- bit SRES provides reasonable protection against a hacker who is trying to decode the value. It also reduces the chance that the PIN code will be compromised by an attacker if the correct SRES value is determined.

Fig. 4 illustrates Bluetooth data encryption between two Bluetooth devices. The ACO, the current link key $\left(\mathrm{K}_{\mathrm{A}}\right.$ or $\left.\mathrm{K}_{\mathrm{AB}}\right)$ and a 128-bit pseudorandom number EN_RAND are inputs to the encryption key generation function $\mathrm{E}_{3}$ that is used for generating an encryption key $\left(\mathrm{K}_{\mathrm{C}}\right)$. The master (device A) generates the EN_RAND and sends it to the slave (device B) via air in an unencrypted form. The $K_{C}$ is produced in both devices using $K_{C}=E_{3}\left(E_{-} \_R A N D_{A}\right.$, ACO, Link key), where the Link key is the $\mathrm{K}_{\mathrm{A}}$ or the $\mathrm{K}_{\mathrm{AB}}$.

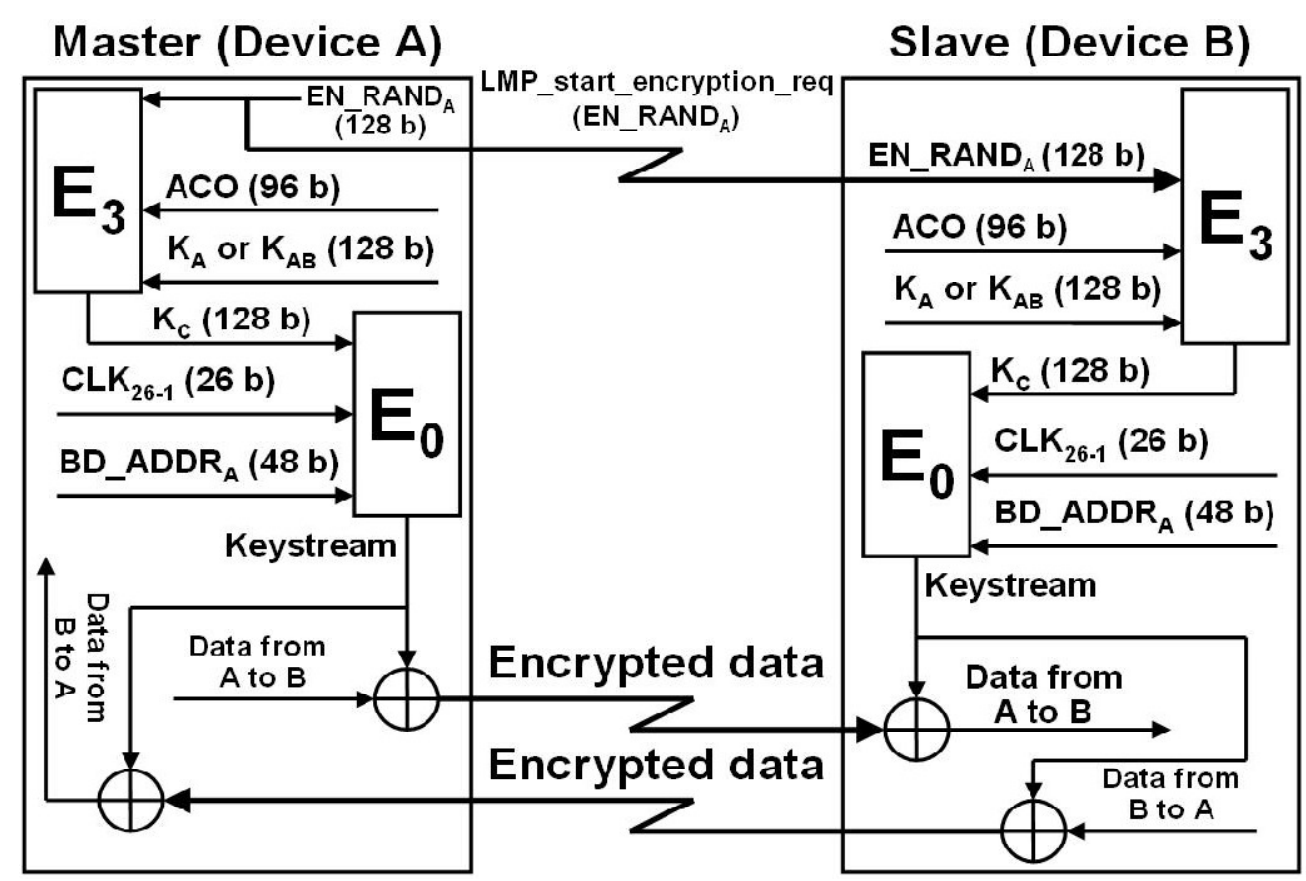

Fig.4 Bluetooth data encryption

The key generator function $\mathrm{E}_{0}$ (Fig. 4) makes symmetric encryption possible by generating the same cipher bit stream, or a key in both devices. The inputs to the $\mathrm{E}_{0}$ function are the $\mathrm{K}_{\mathrm{C}}$, the BD_ADDR of the master $\left(\mathrm{BD}_{-} \mathrm{ADDR} \mathrm{A}_{\mathrm{A}}\right)$, and the 26 bits of the master's real-time clock $\left(\mathrm{CLK}_{26-1}\right)$. The key is generated by the $\mathrm{E}_{0}\left(\mathrm{~K}_{\mathrm{C}}, \mathrm{CLK}_{26-1}, \mathrm{BD} \mathrm{ADDR}_{\mathrm{A}}\right)$ function that is reinitialized for every new sent or received baseband packet, (i.e., the $\mathrm{CLK}_{26-1}$ is updated for every new baseband packet). It means that the inputs to the $\mathrm{E}_{0}$ are used for a lifetime of one baseband packet, and therefore a new key is generated for every new baseband packet.

In Bluetooth versions up to 2.0+EDR, the pairing is based exclusively on the principle that both devices share the same PIN code or passkey. The PIN is the only source of entropy for the 
shared secret. As the PINs often contain only four decimal digits, the strength of the resulting keys is not enough for protection against passive eavesdropping on communication. Even with longer 16-character alphanumeric PINs full protection against active eavesdropping cannot be achieved and Man-In-The-Middle (MITM) attacks on Bluetooth communications can easily break the protection.

The Bluetooth version 2.1+EDR and higher version adds a new specifcation for the pairing procedure called secure simple pairing (SSP). Its main goal is to improve the security of pairing by providing protection against passive eavesdropping and MITM attacks. Instead of using (often short) passkeys as the only source of entropy for building the link keys, SSP employs ECDH public-key cryptography. To construct the link key, devices use public-private key pairs and the Bluetooth addresses of the devices. Passive eavesdropping is effectively blocked by the SSP, as running an exhaustive search on a private key with approximately 95 bits of entropy is currently considered to be infeasible in a short time.

\section{BLUETOOTH NETWORK VULNERABILITIES}

Since there are now billions of Bluetooth devices in use, malicious security violations are common events now and it is expected to increase in the near future. On the contrary, the increased usage of Bluetooth devices makes security concerns even more alarming. Hence, Bluetooth security architecture needs a constant upgrading to prevent new unknown threats. Like any other wireless communication system Bluetooth transmission can be deliberately jammed or intercepted. False or modified information could be passed to the devices by the cyber criminals. Security threats in Bluetooth can be divided into three major categories [15] as follows:

- Disclosure threat: The information can leak fromthe target system to an eavesdropper that is notauthorized to access the information.

- Integrity threat: The information can be deliberately altered to mislead the recipient.

- Denial of Service (DoS) threat: The users can be blocked to get access to a service by making it either unavailable or severely limiting its availability to an authorized user.

Bluetooth security is currently a very active research area in both academia and industry. Security threats like disclosure and integrity attacks typically compromise some sensitive information and therefore, can be very dangerous. On the other hand, DoS attacks typically only annoy Bluetooth network users and are considered to be less dangerous. Powerful directional antennas can be used to considerably increase the scanning, eavesdropping and attacking range of almost any kind of Bluetooth device. One good example of a long-distance attacking tool is the Blue Sniper Rifle. It is a rifle stock with a powerful directional antenna attached to a small Bluetooth-compatible computer. The scanning, eavesdropping and attacking can be done over a mile away from the target devices. Therefore, the possibility that an attacker is using range enhancement equipment for disclosure, integrity and DoS attacks should be taken seriously.

Nowadays, it is also possible to transform a standard Bluetooth dongle into a full-blown Bluetooth Sniffer. Tools for reverse engineering the firmware of Bluetooth dongles are also available. The tools include a disassembler for the official firmware, and an assembler that can be used for writing a custom firmware. With these tools one can now write a custom firmware 
International Journal of Distributed and Parallel Systems (IJDPS) Vol.3, No.1, January 2012

for CSR based Bluetooth dongles to include raw access for Bluetooth sniffing. Moreover, the techniques for finding hidden (i.e., non-discoverable) Bluetooth devices in an average of one minute will be ported onto a standard CSR dongle via a custom firmware. This will open new doors for practical Bluetooth security research and it will also provide a cheap basic weapon to all attackers for Bluetooth sniffing. Therefore, Bluetooth sniffing has become a very popular sport among attackers and hackers. Thus making Bluetooth security becomes even more alarming. There are some other threats that have been reported in the literatures fall outside of these three categories. Some of the threats are presented in the following section.

\section{EXISTING REPORTS OF BLUETOOTH THREATS}

The problems regarding Bluetooth security have been reported since its inception. But, it has not been considered as a significant problem until its adaptation into mobile devices. A brief overview of some of the real incidents is listed below:

- In 2003, Bend and Adam from A.L. Digital Ltd Discovered and published serious flaws in Bluetooth technology regarding the protocol. Their investigations concluded that the security flaws could lead to loss of personal information of a user [15].

- In 2004, the first Bluetooth virus was reported in the literatures as a 'proof-of-concept'. It was proved as a potential threat to the Bluetooth technology [16].

- In January 2005, a mobile malware called 'Lasco' was detected. Lasco was a selfreplicating worm, which was successful in rendering a mobile device unstable before infecting another device [17].

- In April 2005, Cambridge University published a paper documenting actual passive attacks by implementing off-line PIN cracking [18].

- In August 2005, Bluetooth enabled phones were used to track other mobile device left inside of cars [19].

- In April 2006, researchers from Secure Network and F-Secure published a report addressing that a large number of devices were left in a visible state that posed the possibility of spread of a Bluetooth worm [20].

- In October 2007, Kevin Finistere and Thierry Zoller demonstrated the first Bluetooth and link key cracking technique at a conference. A remote root shell via Bluetooth on Mac OS X v10.3.9 and v10.4 was used in that demonstration [21].

Bluetooth devices are exposed to malicious intervention during the process of pairing with another device. These weaknesses are primarily due to flaws in the link key establishment protocol, which is required for devices to pair, and the fact that the encryption of a session is optional and created at the end of the pairing process. It means that the various types of attacks can be performed well before pairing is complete. Even after the pairing is complete, the attackers can still sniff the airwaves to gain enough information to steal link keys so that they can deceptively authenticate or perform Man-in-the-Middle (MITM) attacks to impersonate other devices.

Some other reported attacks on the Bluetooth security are (1) MAC spoofing attack, (2) PIN cracking attack, (3) Man-in-the-Middle/Impersonation attack, (4) BlueJacking attack, (5) BlueSnarfing attack, (6) BlueBugging attack, (7) BluePrinting attack, (8) Blueover attack, (9) 
International Journal of Distributed and Parallel Systems (IJDPS) Vol.3, No.1, January 2012

off-line PIN recovery attack, (10) brute-force attack, (11) reflection attack, (12) backdoor attack, (13) DoS attack, (14) Cabir worm, (15) Skulls worm, and (16) Lasco worm [22-25].

\section{MAC Spoofing Attack}

Among all passive attacks, the most frequently reported attacks are classified as MAC spoofing and PIN cracking attacks. Malicious attackers can perform MAC spoofing during the link key generation while Piconets are being formed. Assuming the attack is made prior to successful pairing and before encryption is established attackers can easily intercept data intended for other devices. Attackers, with specialized hardware, can easily use spoofing to terminate legitimate connections or capture and/or manipulate data while in transit. Bluetooth SIG did not provide a good solution to prevent this type of attack. They only advised the users to do the pairing process in private settings. They also suggested that a long, random, and variable PIN numbers should be used.

\section{PIN Cracking attack}

Using a Bluetooth frequency sniffer (or protocol analyzer) and acquisition of a FHS packet, attackers can attempt to acquire IN_RAND, LK_RAND and the initialization key during the entire pairing and authentication processes. The attacker would have to list all of the possible permutations of the PIN. Using the acquired IN_RAND and BD_ADDR they would need to try possible permutations as input in the $\mathrm{E}_{22}$ algorithm. Eventually they would be able to find the correct initialization key. The next step is to hypothesize and test possibilities of the shared session link key using all of the previous data. Assuming the right information is collected, the proper equipment is used, and enough time is allowed, PIN cracking becomes a fairly simple task. The proposed solutions for these types of attacks involve different pairing and authentication schemes that involves using a combination of public/private keys.

\section{Man-in-the-Middle/Impersonation Attack}

Man-in-the-Middle and impersonation attacks actually involve the modification of data between devices communicating in a Piconet. A Man-in-the-Middle attack involves relaying of authentication message unknowingly between two devices in order to authenticate without knowing the shared secret keys. By forwarding the message of two devices trying to pair, an attacker will relay two unique link keys. By acting between two devices an attacker can trick two devices into believing they are paired when in fact they have paired with the attacker. The suggested solutions to this kind of attack involve incorporating more Piconet specific information into the pairing process. For example, timestamps and nested mutual authentication can be used to determine the legitimacy of a device's challenge before responses are sent in return.

\section{BlueJacking Attack}

Bluejacking is the process of sending unsolicited messages to Bluetooth-enabled devices. This does not involve altering any data from the device, but nonetheless, it is unsolicited. Devices that are set in non-discoverable mode are not susceptible to Bluejacking. In order for Bluejacking to work, the sending and receiving devices must be within 10 meters of each other. While this method has been widely used for promotional purposes, Bluetooth device owners should be careful about not adding the contacts to their address books. Bluejacking is usually not done with malicious intent. Repetitive spam messages can be annoying to the user. In some cases, Bluejacking can render the product inoperable. This can also open the door to a variety of other attacks. 
International Journal of Distributed and Parallel Systems (IJDPS) Vol.3, No.1, January 2012

\section{BlueSnarfing Attack}

Bluesnarfing is a method of hacking into a Bluetooth-enabled mobile phone and copying its entire contact book, calendar or anything else stored in the phone's memory. By setting the device in non-discoverable a user can minimizes the chance of this kind of attack. However, the software tools required to steal information from Bluetooth enabled mobile phones are widely available in the Web. Leading telecommunication giants like Nokia and Sony Ericsson are ensuring that new phones coming to market will not be susceptible to Bluesnarfing attack.

\section{BlueBugging Attack}

A BlueBugging attack means that an attacker connects to the target device (typically a Bluetooth mobile phone), without alerting its owner, and steals some sensitive information. Assuming an attacker has full access to the address translation (AT) command set available in GSM (Global System for Mobile ) an attacker can exploit the AT commands. It means that the attacker can, in addition to stealing information, send text messages to premium numbers. Hence the attacker can initiate phone calls to premium numbers, write to phonebook entries, connect to the Internet, set call forwards, try to slip a Bluetooth virus or worm to the target device.

\section{BluePrinting Attack}

A BluePrinting attack is used to determine the manufacturer, device model and firmware version of the target device. An attacker can use Blueprinting to generate statistics about Bluetooth device manufacturers and models, and to find out whether there are devices in the range of vulnerability that have issued with Bluetooth security, for example. BluePrint 0.1 is a tool for performing BluePrinting attack. It runs on Linux and it is based on the BlueZ protocol stack. BluePrinting attacks work only when the BD_ADDR of the target device is known.

\section{Blueover attack}

Blueover and its successor Blueover II are derived from Bluetooth. However, because they run on handheld devices such as PDAs or mobile phones and are capable of stealing sensitive information by using a BlueBugging attack. A Blueover attack can be done secretly, by using only a Bluetooth mobile phone with Blueover or Bluover II installed. Bluleover and Bluover II run on almost every J2ME (Java 2 Micro Edition) compatible handheld device. They are intended to serve as auditing tools which can be used for checking whether Bluetooth devices are vulnerable or not, but they can be used for attacking against Bluetooth devices as well. A Blueover attack is dangerous only if the target device is vulnerable to BlueBugging. Moreover, an attacker has to know the BD_ADDR of the target device.

\section{Off-Line PIN Recovery Attack}

An off-line PIN recovery attack is based on intercepting the IN_RAND value, LK_RAND values, AU_RAND value and SRES value, and after that trying to calculate the correct SRES value by guessing different PIN values until the calculated SRES equals the intercepted SRES. It is worth noting that SRES is only 32 bits long. Therefore, a SRES match does not necessarily guarantee that an attacker has discovered the correct PIN code, but the chances are quite high especially if the PIN code is short.

\section{Brute-Force Attack}

A brute-force BD_ADDR scanning attack uses a brute-force method only on the last three bytes of a BD_ADDR, because the first three bytes are publicly known and can be set as fixed. A 
International Journal of Distributed and Parallel Systems (IJDPS) Vol.3, No.1, January 2012

brute-force BD_ADDR scanning attack is perhaps the most feasible attack when target devices are Bluetooth mobile phones, because millions of vulnerable Bluetooth mobile phones are used every day all over the world.

\section{Reflection Attack}

Reflection attacks (also referred to as relay attacks) are based on the impersonation of target devices. An attacker does not have to know any secret information, because the attacker only relays (reflects) the received information from one target device to another during the authentication. Hence a reflection attack in Bluetooth can be seen as a type of a MITM attack against authentication, but not against encryption. The only information needed is the BD_ADDRs of the target devices.

\section{Backdoor Attack}

The backdoor attack involves establishing a trust relationship through the pairing mechanism, but ensuring that it no longer appears in the target's register of paired devices. In this way, unless the owner is actually monitoring their devices at that moment, a connection is established. The attacker may continue using the resources that a trusted relationship with that device grants access to until the users notice such attacks. The attacker can not only retrieve data from the phone, but other services such as modems, Internet, WAP and GPRS gateways may be accessed without the owner's knowledge or consent. A backdoor attack works only if the BD_ADDR of the target device is known. Moreover, the target device has to be vulnerable to a backdoor attack.

\section{DoS Attacks}

The DoS threats can be roughly divided into two parts: (1) attacks against the physical (PHY) layer, and (2) attacks against protocols above the PHY layer. At the PHY layer, an attacker can jam the Piconet entirely or capture the channel from the legitimate Piconet device. A jammer can disrupt the PHY layer by hopping along with the Piconet devices and send random data in every timeslot. Some typical DoS attacks are described below:

- BD_ADDR duplication attack:An attacker places a 'bug' in the range of the Bluetooth device. The bug duplicates the BD_ADDR of the target device. When any Bluetooth device tries to make a connection with the target device, either the target device or both devices (i.e., the target device and the bug) will respond and jam each other. In this way, the attacker can cause denial of access from the legitimate device. The most effective way to perform this attack is to duplicate the BD_ADDR of the Piconet master device, because all information within the Piconet goes through the master device.

- SCO/eSCO attack: It is based on a real-time two-way voice. It reserves a great deal of a Bluetooth Piconet's attention so that the legitimate Piconet devices are not allowed to get the service within a reasonable period of time. The most effective way to perform this type of attack is to establish a SCO or an e-SCO link with the Piconet master.

- Big NAK attack: It is based on the idea of putting the target device on an endless retransmission loop so that the legitimate Piconet devices have considerably slowed throughput. In this attack, an attacker requests any information from the target device and each time the requested information is received, the attacker sends Negative Acknowledgement (NAK). Hence, the target device keeps sending the requested information again and again. 
- L2CAP Guaranteed Service attack: An attacker requests the highest possible data rate or the smallest possible latency from the target device so that all other connections are refused, and the throughput is reserved for the attacker.

- Battery exhaustion attack: It is based on the idea of occupying the target device in such a way that it consumes rather quickly the battery life of the target device.

\section{Cabir worm}

The Cabir worm is a kind of malicious software that uses Bluetooth technology to seek out available Bluetooth devices and sends itself to them. The Cabir worm currently only affects mobile phones that use the Symbian series 60 user interface platform. Furthermore, the user has to manually accept the worm and install the malware in order to infect the phone. It is usually done by disguising the Cabir worm impersonating another application and the user is unaware of it. The Cabir worm shows that it is achievable to write mobile viruses that spread via Bluetooth and may cause other hackers to explore the possibilities of writing Bluetooth viruses. The Mabir worm is essentially a variant of the Cabir worm that uses Bluetooth and Multimedia Messaging Service messages (MMS) to replicate.

\section{Skulls worm}

Skulls.D (also referred to as SymbOS/Skulls.D) is a malicious SIS (Symbian Installation System) trojan file that pretends to be Macromedia Flash player for Symbian mobile phones which support the Series 60 platform. It arrives in the target mobile phone via Bluetooth in a similar way that Cabir follows. When the user opens the SIS file and chooses to install it, the SymbOS/Cabir.M worm (i.e., a variation of the Cabir worm) will be installed in the target mobile phone. Both the system applications and the third party applications needed to disinfect viruses and worms will be disabled. An animation showing a flashing skull picture will also be displayed on the background of the target device's display at the time of using the application by the user. When the worm is activated, it immediately starts searching for new Bluetooth devices to infect.

\section{Lasco Worm}

Lasco (also referred to as SymbOS/Lasco.A or EPOC/Lasco.A) is a Bluetooth worm and a SIS file infecting virus running in Symbian mobile phones which support the Series 60 platform. It arrives in the target mobile phone via Bluetooth in a similar way as Cabir and Skulls.D do. When the user opens the velasco.sis file and chooses to install it, the worm will be activated and it will immediately starts searching for new Bluetooth devices to infect. In addition to sending itself via Bluetooth, it is also capable of inserting itself into other SIS files in the target device. Therefore, if infected SIS files are copied to another device, Lasco worm will also affect the other device too.

\section{COUNTER MEASURES}

As technology makes progress, new attacks are being developed by the attackers. It is not possible to take counter measures against all the weaknesses and the security holes of Bluetooth. The weakest part of the Bluetooth technology involves the pairing process in which it establishes trusted relationships with other devices. Table 2 below provides an overview of some of the known security vulnerabilities with Bluetooth communication [26]. 
International Journal of Distributed and Parallel Systems (IJDPS) Vol.3, No.1, January 2012

Table 2: Bluetooth Security Vulnerabilities

\begin{tabular}{|c|c|c|}
\hline & Security Vulnerabilities & Description \\
\hline \multicolumn{3}{|c|}{ Versions Before Bluetooth v1.2 } \\
\hline 1 & $\begin{array}{l}\text { Unit key is reusable and } \\
\text { becomes public once used. }\end{array}$ & $\begin{array}{l}\text { A unit key should be used as input to generate a random } \\
\text { key. A key set should be used instead of only one unit } \\
\text { key. }\end{array}$ \\
\hline 2 & $\begin{array}{l}\text { Unit key sharing can lead to } \\
\text { eavesdropping. }\end{array}$ & $\begin{array}{l}\text { Attacker may be able to compromise the security between } \\
\text { two users if the attacker has communicated with either of } \\
\text { the other two users. This is because the link key (unit } \\
\text { key), derived from shared information has been } \\
\text { disclosed. }\end{array}$ \\
\hline \multicolumn{3}{|c|}{ Versions Before Bluetooth v2.1 } \\
\hline 3 & Short PINs are allowed. & $\begin{array}{l}\text { Weak PINs, which are used for the generation of link and } \\
\text { encryption keys, can be easily cracked. People have a } \\
\text { tendency to select short PINs. }\end{array}$ \\
\hline 4 & PIN management is lacking. & $\begin{array}{l}\text { Establishing use of adequate PINs in an enterprise setting } \\
\text { with many users may be difficult. Scalability problems } \\
\text { frequently yield security problems. }\end{array}$ \\
\hline 5 & $\begin{array}{l}\text { Encryption keystream repeats } \\
\text { after } 23.3 \text { hours of use to keep } \\
\text { the connection alive. }\end{array}$ & $\begin{array}{l}\text { The encryption keystream is dependent on the link key, } \\
\text { EN_RAND,Master BD_ADDR, and Clock. Only the } \\
\text { Master's clock will change during a particular encrypted } \\
\text { connection. If a connection lasts for more than } 23.3 \\
\text { hours, the clock value will begin to repeat, hence } \\
\text { generating an identical keystream to that used earlier in } \\
\text { the connection. }\end{array}$ \\
\hline \multicolumn{3}{|c|}{ All Versions } \\
\hline 6 & $\begin{array}{l}\text { Link keys are stored } \\
\text { improperly. }\end{array}$ & $\begin{array}{l}\text { Link keys can be read or modified by an attacker if they } \\
\text { are not securely stored and protected via access codes. }\end{array}$ \\
\hline 7 & $\begin{array}{l}\text { Attempts for authentication are } \\
\text { repeated. }\end{array}$ & $\begin{array}{l}\text { A limiting feature needs to be incorporated in the } \\
\text { specification to prevent unlimited requests. The } \\
\text { Bluetooth specification currently requires a time-out } \\
\text { period between repeated attempts that will increase } \\
\text { exponentially. }\end{array}$ \\
\hline 8 & $\begin{array}{l}\text { Strength of the challenge- } \\
\text { response pseudo-random } \\
\text { generator is not known. }\end{array}$ & $\begin{array}{l}\text { The Random Number Generator (RNG) may produce } \\
\text { static number or periodic numbers that may reduce the } \\
\text { effectiveness of the authentication scheme. }\end{array}$ \\
\hline 9 & $\begin{array}{l}\text { Encryption key length is } \\
\text { negotiable. }\end{array}$ & $\begin{array}{l}\text { The specification allows devices to negotiate encryption } \\
\text { keys as small as one byte. A more robust encryption key } \\
\text { generation procedure needs to be incorporated. }\end{array}$ \\
\hline 10 & The master key is shared. & $\begin{array}{l}\text { A better broadcast keying scheme needs to be } \\
\text { incorporated into the specification. }\end{array}$ \\
\hline 11 & No user authentication exists. & $\begin{array}{l}\text { Only device authentication is provided by the } \\
\text { specification. Application level security, including user } \\
\text { authentication, can be added via overlay by the } \\
\text { application developer. }\end{array}$ \\
\hline 12 & $\begin{array}{l}\text { The } E_{0} \text { stream cipher algorithm } \\
\text { used for Bluetooth encryption is }\end{array}$ & More robust encryption needs to be incorporated in the \\
\hline
\end{tabular}


International Journal of Distributed and Parallel Systems (IJDPS) Vol.3, No.1, January 2012

\begin{tabular}{|c|l|l|}
\hline & weak. & specification. \\
\hline 13 & $\begin{array}{l}\text { Privacy may be compromised if } \\
\text { the Bluetooth device addres } \\
\text { (BD_ADDR) is captured and } \\
\text { associated with a particular } \\
\text { user. }\end{array}$ & $\begin{array}{l}\text { Once the BD_ADDR is associated with a particular user, } \\
\text { that user's activities could be logged, resulting in a } \\
\text { breach of privacy. }\end{array}$ \\
\hline 14 & $\begin{array}{l}\text { Device authentication is simple } \\
\text { shared-key challenge-response. }\end{array}$ & $\begin{array}{l}\text { One-way-only challenge-response authentication is } \\
\text { subject to MITM attacks. Bluetooth provides for mutual } \\
\text { authentication, which should be used to provide } \\
\text { verification that users are legitimate. }\end{array}$ \\
\hline 15 & $\begin{array}{l}\text { End-to-end security is not } \\
\text { performed. }\end{array}$ & $\begin{array}{l}\text { Only individual links are encrypted and authenticated. } \\
\text { Data is decrypted at intermediate points. End-to-end } \\
\text { security on top of the Bluetooth stack can be provided by } \\
\text { the use of additional security controls. }\end{array}$ \\
\hline 16 & $\begin{array}{l}\text { Security services are limited. } \\
\text { Audit, non-repudiation, and other services are not part of } \\
\text { the standard. These services can be incorporated in an } \\
\text { overlay fashion by the application developer. }\end{array}$ \\
\hline 17 & $\begin{array}{l}\text { Discoverable and connectable } \\
\text { devices are prone to attack. }\end{array}$ & $\begin{array}{l}\text { Any device that must go into discoverable or connectable } \\
\text { mode to pair should only do so for a minimal amount of } \\
\text { time. A device should never be in discoverable or } \\
\text { connectable mode all the time. }\end{array}$ \\
\hline
\end{tabular}

\section{RISK MITIGATION}

Risk mitigation can be achieved in Bluetooth systems by applying countermeasures to address specific threats and vulnerabilities. Some of these countermeasures cannot be achieved through the security features built into the Bluetooth specifications. The countermeasures recommended in the Table 2 do not guarantee a secure Bluetooth environment and cannot prevent all attacks. It should be noted that the development of improved security comes at a cost-financial expenses related to security equipment, maintenance, and operation, which should also be considered during development of new security features.

The first line of defense is to provide an adequate level of knowledge and understanding for the users of Bluetooth-enabled devices. Users should understand the security policies that address the use of Bluetooth enabled devices and their own responsibilities. The Bluetooth security experts should include awareness based education to support user's understanding and knowledge of Bluetooth security. Policy documents should include a list of approved uses for Bluetooth, and the type of information that may be transferred over Bluetooth networks. The security policy should also specify a proper password usage scheme. Most users do not pay attention while assigning strong pass codes because most of them are not aware of the proper techniques.

The general nature and mobility of Bluetooth enabled devices increases the difficulty of employing traditional security measures. Nevertheless, a number of countermeasures can be enacted to secure Bluetooth devices and communications, ranging from distance and power output to general operation practices. 
International Journal of Distributed and Parallel Systems (IJDPS) Vol.3, No.1, January 2012

Table 3 provides a Bluetooth security measure with recommendations for creating and maintaining secure Bluetooth Piconets. These recommendations are applicable for most of the Bluetooth profiles [10] that requires information exchange over Piconets. Note that some commercially available Bluetooth devices cannot be configured to meet the recommendations as they do not provide encryption and often use a four-digit PIN with a default value like "0000" that cannot be changed.

Table 3: Bluetooth Security Countermeasures

\begin{tabular}{|c|c|c|}
\hline \multicolumn{2}{|r|}{ SECURITY RECOMMENDATION } & JUSTIFICATION \\
\hline 1 & $\begin{array}{l}\text { Ensure that Bluetooth users are made aware } \\
\text { of their security-related responsibilities } \\
\text { regarding Bluetooth use. }\end{array}$ & $\begin{array}{l}\text { A security awareness program helps } \\
\text { users to follow security practices that } \\
\text { help prevent security loopholes. }\end{array}$ \\
\hline 2 & $\begin{array}{l}\text { Change the default settings of the Bluetooth } \\
\text { device to reflect optimal security standard }\end{array}$ & $\begin{array}{l}\text { Because default settings are generally not } \\
\text { secured, a careful review should be } \\
\text { performed for optimal settings. }\end{array}$ \\
\hline 3 & $\begin{array}{l}\text { Set Bluetooth devices to the lowest necessary } \\
\text { and sufficient power level so that } \\
\text { transmissions remain within the secure } \\
\text { perimeter of the desired network range. }\end{array}$ & $\begin{array}{l}\text { Setting Bluetooth devices to the lowest } \\
\text { necessary and sufficient power level } \\
\text { ensures a secure range of access to } \\
\text { authorized users }\end{array}$ \\
\hline 4 & $\begin{array}{l}\text { Choose PIN codes that are sufficiently } \\
\text { random and long. Avoid static and weak } \\
\text { PINs, such as PINs containing all 0's or 1's. }\end{array}$ & $\begin{array}{l}\text { PIN codes should be random so that they } \\
\text { cannot be easily guessed by attackers. } \\
\text { Longer PIN codes are more resistant to } \\
\text { brute force attacks. The use of a fixed } \\
\text { PIN is not acceptable for connections. }\end{array}$ \\
\hline 5 & $\begin{array}{l}\text { Ensure that the link keys are based on } \\
\text { combination keys rather than unit keys. }\end{array}$ & $\begin{array}{l}\text { The use of shared unit keys can lead to } \\
\text { successful MITM attacks. So, using } \\
\text { combination keys are safer. }\end{array}$ \\
\hline 6 & $\begin{array}{l}\text { For devices using Secure Simple Pairing } \\
\text { (SSP), avoid using the "Just Works" model. }\end{array}$ & $\begin{array}{l}\text { The "Just Works" association model } \\
\text { does not provide MITM protection. } \\
\text { Devices that only support "Just Works" } \\
\text { should be avoided for confidential } \\
\text { communications. }\end{array}$ \\
\hline 7 & $\begin{array}{l}\text { Service and profile lockdown of device } \\
\text { Bluetooth stacks should be performed }\end{array}$ & $\begin{array}{l}\text { Many Bluetooth stacks are designed to } \\
\text { support multiple profiles and associated } \\
\text { services. The Bluetooth stack on a device } \\
\text { should be locked down to ensure only } \\
\text { approved profiles and services are } \\
\text { available for use. }\end{array}$ \\
\hline 8 & $\begin{array}{l}\text { Bluetooth devices should be configured by } \\
\text { default to be undiscoverable except as needed } \\
\text { for pairing. }\end{array}$ & $\begin{array}{l}\text { Bluetooth interfaces should be } \\
\text { configured as non-discoverable, which } \\
\text { prevents visibility to other Bluetooth } \\
\text { devices except when discovery is } \\
\text { specifically needed. Also, the default } \\
\text { self-identifying or discoverable names } \\
\text { provided on Bluetooth devices should be } \\
\text { changed to anonymous and } \\
\text { unidentifiable names. }\end{array}$ \\
\hline 9 & Use Link Encryption for all Bluetooth & Link encryption should be used to secure \\
\hline
\end{tabular}


International Journal of Distributed and Parallel Systems (IJDPS) Vol.3, No.1, January 2012

\begin{tabular}{|c|c|c|}
\hline & $\begin{array}{l}\text { connections (i.e., do not use "non-secure" } \\
\text { mode). }\end{array}$ & $\begin{array}{l}\text { all data transmissions during a Bluetooth } \\
\text { connection; otherwise transmitted data is } \\
\text { vulnerable to eavesdropping. }\end{array}$ \\
\hline 10 & $\begin{array}{l}\text { If multi-hop wireless communication is being } \\
\text { utilized, ensure that encryption is enabled on } \\
\text { every link in the communication chain. }\end{array}$ & $\begin{array}{l}\text { Every link should be secured because } \\
\text { one unsecured link results in } \\
\text { compromising the entire communication } \\
\text { chain. }\end{array}$ \\
\hline 11 & $\begin{array}{l}\text { Ensure device mutual authentication is } \\
\text { performed for all accesses. }\end{array}$ & $\begin{array}{l}\text { Mutual authentication is required to } \\
\text { provide verification that all devices on } \\
\text { the network are legitimate. }\end{array}$ \\
\hline 12 & $\begin{array}{l}\text { Enable encryption for all broadcast } \\
\text { transmissions }\end{array}$ & $\begin{array}{l}\text { Broadcast transmissions secured by link } \\
\text { encryption provide a layer of security } \\
\text { that protects these transmissions from } \\
\text { user interception for malicious purposes. }\end{array}$ \\
\hline 13 & $\begin{array}{l}\text { Configure encryption key sizes to the } \\
\text { maximum allowable limit. }\end{array}$ & $\begin{array}{l}\text { Maximum allowable key sizes provide } \\
\text { protection from brute force attacks. }\end{array}$ \\
\hline 14 & $\begin{array}{l}\text { Establish a "minimum key size" for any key } \\
\text { negotiation process. }\end{array}$ & $\begin{array}{l}\text { Establishing minimum key sizes ensures } \\
\text { that all keys are long enough to be } \\
\text { resistant to brute force attacks. } \\
\text { Preferably, keys should be at least } 128 \\
\text { bits long. }\end{array}$ \\
\hline 15 & $\begin{array}{l}\text { Ensure that Bluetooth devices are turned off } \\
\text { when they are not used. }\end{array}$ & $\begin{array}{l}\text { Bluetooth capabilities should be disabled } \\
\text { on all Bluetooth devices, except when } \\
\text { the user explicitly enables Bluetooth to } \\
\text { establish a connection. Shutting down } \\
\text { Bluetooth devices (when not in use) } \\
\text { minimizes exposure to potential } \\
\text { malicious activities. }\end{array}$ \\
\hline 16 & $\begin{array}{l}\text { Perform pairing as infrequently as possible, } \\
\text { ideally in a secure area where attackers cannot } \\
\text { realistically observe the pass key entry and } \\
\text { intercept Bluetooth pairing messages. (Note: } \\
\text { A "secure area" is defined as a non-public } \\
\text { area). Users should not respond to any } \\
\text { messages requesting a PIN, unless the user } \\
\text { has initiated a pairing process. }\end{array}$ & $\begin{array}{l}\text { Pairing is a vital security function and } \\
\text { requires that users maintain a security } \\
\text { awareness of possible eavesdroppers. If } \\
\text { an attacker can capture the transmitted } \\
\text { frames associated with pairing, } \\
\text { determining the link key is } \\
\text { straightforward }\end{array}$ \\
\hline 17 & $\begin{array}{l}\text { A service-level security mode (i.e. Security } \\
\text { Mode } 2 \text { or 4) should only be used in a } \\
\text { controlled and well-understood environment. }\end{array}$ & $\begin{array}{l}\text { Security Mode } 3 \text { provides link-level } \\
\text { security prior to link establishment, while } \\
\text { Security Modes } 2 \text { and } 4 \text { allow link-level } \\
\text { connections before any authentication or } \\
\text { encryption is established. It is highly } \\
\text { recommended that devices use Security } \\
\text { Mode 3. }\end{array}$ \\
\hline 18 & $\begin{array}{l}\text { In the event that a Bluetooth device is lost or } \\
\text { stolen, users should immediately un-pair the } \\
\text { missing device from all other Bluetooth } \\
\text { devices with which it was previously paired. }\end{array}$ & $\begin{array}{l}\text { This will prevent an attacker from using } \\
\text { the lost or stolen device to access another } \\
\text { Bluetooth device owned by the user. }\end{array}$ \\
\hline 19 & $\begin{array}{l}\text { This will prevent an attacker from using the } \\
\text { lost or stolen device to access another } \\
\text { Bluetooth device owned by the user. }\end{array}$ & $\begin{array}{l}\text { Antivirus software should be installed on } \\
\text { frequently targeted Bluetooth-enabled } \\
\text { hosts to ensure that known malware is }\end{array}$ \\
\hline
\end{tabular}


International Journal of Distributed and Parallel Systems (IJDPS) Vol.3, No.1, January 2012

\begin{tabular}{|l|l|l|}
\hline & & not introduced to the Bluetooth network. \\
\hline 20 & $\begin{array}{l}\text { Users should not accept transmissions of any } \\
\text { kind from unknown or suspicious devices. } \\
\text { These types of transmissions include } \\
\text { messages, files, and images. }\end{array}$ & $\begin{array}{l}\text { With the increase in the number of } \\
\text { Bluetooth-enabled devices, it is } \\
\text { important that users only establish } \\
\text { connections with other trusted devices } \\
\text { and only accept content from these } \\
\text { trusted devices }\end{array}$ \\
\hline
\end{tabular}

\section{Conclusion}

This paper presented an overview of some of the major attacks that Bluetooth has faced over the years along with some possible solutions. Some safety tips for the users have also been provided to instantly create awareness among them to be more cautious about their personal information. Although a vast majority of devices now communicate using this technology, the risks are far greater if the security threats are overlooked by our peers in this industry. Bluetooth security specialists need to provide automatic updates to its security protocols and user privacy protection methods for every new security breach so that protection of the device user's personal information becomes the primary objective. Due to limitations in time and resources, only a comprehensive literature survey has been presented in this paper. Emerging devices all have Bluetooth as a mandatory feature and its potential applications are increasing, so its future vulnerabilities needs to be explored through further research in this field. The bottom line is, we need technology to survive and technology needs us to evolve ensuring our safety first.

\section{REFERENCES}

[1] "The Bluetooth Blues", available at http://www.information-age.com/article/2001/may/the_bluetooth_blues

[2] Bluetooth SIG, Specification of the Bluetooth System: Volume 2, Profile, Version 1.1, Feb. 22, 2001. available at:

https://www.bluetooth.org/About/bluetooth_sig.htm

[3] "The History of Bluetooth", available at:

http://www.bluetomorrow.com/about-bluetoothtechnology/history-of-bluetooth/bluetoothhistory.html

[4] Monson, Heidi - "Bluetooth Technology and Implications" available at:

http://www.sysopt.com/features/ network/article.php/3532506 (1999-12- 14).

[5] "How Bluetooth Works", available at:

http://en.kioskea.net/contents/bluetooth/bluetooth-fonctionnement.php3.

[6] Mohammed Mana, Mohammed Feham, and Boucif Amar Bensaber, "A light weight protocol to provide location privacy in wireless body area networks", International Journal of Network Security and its Applications (IJNSA), Vol.3, No.2, March 2011 
International Journal of Distributed and Parallel Systems (IJDPS) Vol.3, No.1, January 2012

[7] Yasir Arfat Malkani and Lachhman Das Dhomeja, "PSim: A tool for analysis of device pairing methods", International Journal of Network Security \& Its Applications (IJNSA), Vol.1, No.3, October 2009

[8] Kumar, A., et al. Caveat eptor, "A comparative study of secure device pairing methods", IEEE International Conference on Pervasive Computing and Communications (PerCom-09). 2009.

[9] Jochen Schiller, "Mobile Communications", Second Edition, Addison Wesley Publications, 2003, pp. 290-292

[10] Bluetooth Profiles" Bluetooth Resource Center, Palowireless Pty Ltd. available at: http://www.palowireless.com/ infotooth/tutorial/profiles.asp

[11] SaileshRathi, "Bluetooth Protocol Architecture", Microware Systems Corporation available at: http://www.dedicated-systems.com/Magazine/00q4/2000q4_p028.pdf

[12] "Bluetooth Versions", summary of all the Bluetooth versions released to date, available at: http://www.bluetomorrow.com/about-bluetooth-technology/general-bluetoothinformation/bluetooth-versions.html

[13] Bluetooth Version 4.0 Released. Bluetooth SIG, available at: http://www.bluetooth.com/Pages/High-Speed.aspx

[14] Keijo Haataja, "Security Threats and Countermeasures in Bluetooth Enabled Systems", Kuopio University Library, 2009, pp. 55-62

[15] "The BlueBug", a Bluetooth virus, available at: http://trifinite.org/trifinite_stuff_bluebug.html

[16] John Oates, "Virus attacks mobiles via Bluetooth", available at: http://www.theregister.co.uk/2004/06/15/symbian_virus/

[17] F-Secure Article on Lasco.A Worm, available at: http://www.f-secure.com/v-descs/lasco_a.shtml

[18] Ford-Long Wong, Frank Stajano, Jolyon Clulow, "Repairing the Bluetooth pairing protocol". University of Cambridge Computer Laboratory, available at: http://www.cl.cam.ac.uk/research/dtg/ fw242/publications/2005-WongStaClu-bluetooth.pdf

[19] Phone pirates in seek and steal mission", Cambridge Evening News, available at: http://www.cambridge-news.co.uk/news/region_wide/2005/08/17/

[20] "Going Around with Bluetooth in Full Safety", available at: http://www.securenetwork.it/ricerca/whitepaper/download/bluebag_brochure.pdf 
International Journal of Distributed and Parallel Systems (IJDPS) Vol.3, No.1, January 2012

[21] Yaniv Shaked, Avishai Wool, "Cracking the Bluetooth PIN" School of Electrical Engineering Systems, Tel Aviv University, available at:

http://www.eng.tau.ac.il/ yash/shaked-wool-mobisys05/

[22] Keijo Haataja, "Security Threats and Countermeasures in Bluetooth-Enabled Systems", Kuopio University Library, 2009, pp. 68-80

[23] Colleen Rhodes, “Bluetooth Security”, East Carolina University, pp.6-9

[24] Karen Scarfone and John Padgette, (Bluetooth Threats) "Guide to Bluetooth Security", Computer Security Division - National Institute of Standards and Technology, US Department of Commerce, 2008, pp. 25-26

[25] Raquel Hill and Billy Falotico, "Bluetooth Wireless Technology Security Threats and Vulnerabilities”, Indiana University Bloomington, 2008, pp. 7-8

[26] Karen Scarfone and John Padgette, (Bluetooth Vulnerabilities) "Guide to Bluetooth Security", Computer Security Division - National Institute of Standards and Technology, US Department of Commerce, 2008, pp.24-25

\section{Authors}

Nateq Be-Nazir Ibn Minar is a Research Scientist currently rendering his expertise independently to institutions and manufacturing industries. He obtained his Bachelor of Science degree in Electrical and Electronics Engineering, with a special interest in Robotics and Industrial Automation, from American International University - Bangladesh in 2008 and later obtained his Master of Science degree, in Telecommunication Engineering, focusing on Wireless Networks. His research interests include satellite communications, network security, low-powered wireless devices, industrial automation, robotics and green technology. He is the CEO of EcoWave - a company dedicated to produce green electronic products for the benefit of the environment. He is an active member of IEEE and does volunteer work for

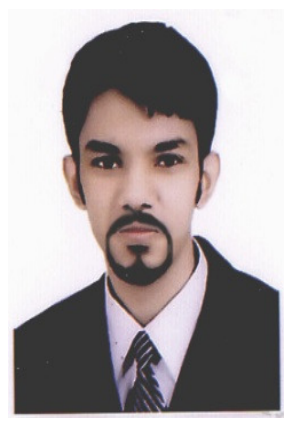
several societies.

Mohammed Tarique is an Assistant Professor in the Department of Electronic and Communication, Ajman University of Science and Technology, in United Arab Emirates. His research interests include Adhoc Networks and wireless network security, network testing and simulations. His research primarily focuses on the design of Wireless Adhoc Networks.

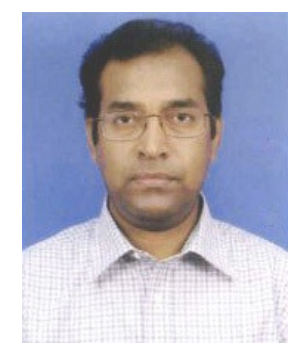

